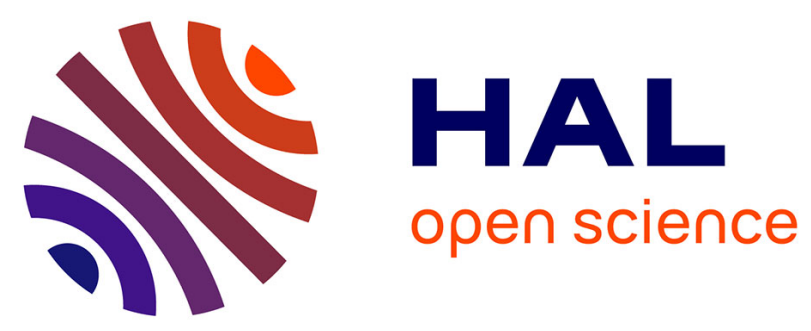

\title{
Maximum Shear Modulus Prediction by Marchetti Dilatometer Test Using Neural Networks
}

\author{
Manuel Cruz, Jorge M. Santos, Nuno Cruz
}

\section{To cite this version:}

Manuel Cruz, Jorge M. Santos, Nuno Cruz. Maximum Shear Modulus Prediction by Marchetti Dilatometer Test Using Neural Networks. 12th Engineering Applications of Neural Networks (EANN 2011) and 7th Artificial Intelligence Applications and Innovations (AIAI), Sep 2011, Corfu, Greece. pp.335-344, 10.1007/978-3-642-23957-1_38. hal-01571370

\author{
HAL Id: hal-01571370 \\ https://hal.inria.fr/hal-01571370
}

Submitted on 2 Aug 2017

HAL is a multi-disciplinary open access archive for the deposit and dissemination of scientific research documents, whether they are published or not. The documents may come from teaching and research institutions in France or abroad, or from public or private research centers.
L'archive ouverte pluridisciplinaire HAL, est destinée au dépôt et à la diffusion de documents scientifiques de niveau recherche, publiés ou non, émanant des établissements d'enseignement et de recherche français ou étrangers, des laboratoires publics ou privés. 


\title{
Maximum Shear Modulus Prediction by Marchetti Dilatometer Test Using Neural Networks.
}

\author{
Manuel Cruz ${ }^{1,2}$, Jorge M. Santos ${ }^{1,2}$, Nuno Cruz ${ }^{3,4}$ \\ 1 ISEP - Instituto Superior de Engenharia do Porto, Portugal \\ 2 LEMA - Laboratório de Engenharia Matemática, Porto \\ 3 Mota-Engil, Portugal \\ 4 Universidade de Aveiro, Portugal \\ emails: mbc,jms (@isep.ipp.pt),nbdfcruz@gmail.com
}

\begin{abstract}
The use of Neural Networks for modeling systems has been widespread, in particular within areas where the great amount of available data and the complexity of the systems keeps the problem very unfriendly to treat following traditional data analysis methodologies. In the last two decades, small strain shear modulus became one of the most important geotechnical parameters to characterize soil stiffness. Finite element analysis have shown that in-situ stiffness of soils and rocks is much higher than was previously thought, and that stress-strain behaviour of these materials is non-linear in most cases with small strain levels, especially in the ground around retaining walls, foundations and tunnels typically in the order of $10^{-2}$ to $10^{-4}$ of strain. Although the best approach seems to be based in measuring seismic wave velocities, deriving the parameter through correlations with in-situ tests is usually considered very useful for design practice. In this work, a new approach using Neural Networks is proposed for sedimentary soils and the results are discussed and compared with some of the most common available methodologies for this evaluation.
\end{abstract}

\section{Introduction}

Maximum shear modulus, $G_{0}$, has been increasingly introduced in stiffness evaluations for design purposes, for the last 10-15 years. At the present moment, one of the best ways for measuring it is to evaluate compression and shear wave velocities and thus obtain results supported by theoretical interpretations. The advantages of this approach are widely known, mainly because test readings are taken through intact soil in its in-situ stress and saturation levels, thus practically undisturbed, and also dynamic stiffness can be close to operational static values $[1,2]$. However, the use of seismic measures implies a specific test and so, many atempts have been made to correlate other in-situ test parameters, such as those obtained by Standard Penetration Test, SPT [3], Piezocone Test, CPTu [4] or Marchetti Dilatometer Test, DMT [5-7] with G0, using cross calibrations (e.g.,seismic or triaxial among others). 
The DMT test is one of the most appropriated for this task (although some relations can be settled using SPT or $\mathrm{CPTu}$ ) since it uses a measurement of a load range related with a specific displacement, which can be used to deduce highly accurate stress-strain relationship $\left(E_{D}\right)$, supported by Theory of Elasticity. Moreover, the type of soil can be numerically represented by DMT Material Index, $I_{D}$, while in situ density, overconsolidation ratio $(\mathrm{OCR})$ and cementation influences can be represented by lateral stress index, $K_{D}$, allowing for high quality calibration of the basic stress-strain relationship [7].

The present paper is organized as follows: In Section 2 an overview of the most important correlations used in this subject is reported, in order to get a clear view of the problem context. In Section 3 the data set is presented, as well as the methodology used for the Neural Networks application on this subject and its results and subsequent discussion. As usual, the last section will be used for conclusions and final remarks.

\section{$2 \quad G_{0}$ prediction by DMT}

Marchetti dilatometer test or flat dilatometer, commonly designated by DMT, has been increasingly used and it is one of the most versatile tools for soil characterization, namely loose to medium compacted granular soils and soft to medium clays, or even stiffer if a good reaction system is provided. The test was developed by Silvano Marchetti [5] and can be seen as a combination of both Piezocone and Pressuremeter tests with some details that really makes it a very interesting test available for modern geotechnical characterization [7]. The main reasons for its usefulness deriving geotechnical parameters are related to the simplicity (no need of skilled operators) and the speed of execution (testing a $10 \mathrm{~m}$ deep profile takes around 1 hour to complete) generating quasi-continuous data profiles of high accuracy and reproducibility.

In its essence, dilatometer is a stainless steel flat blade $(14 \mathrm{~mm}$ thick, $95 \mathrm{~mm}$ wide and $220 \mathrm{~mm}$ length) with a flexible steel membrane (60 $\mathrm{mm}$ in diameter) in one of its faces. The blade is connected to a control unit on the ground surface by a pneumatic-electrical cable that goes inside the position rods, ensuring electric continuity and the transmission of the gas pressure required to expand the membrane. The gas is supplied by a connected tank/bottle and flows through the pneumatic cable to the control unit equipped with a pressure regulator, pressure gauges, an audio-visual signal and vent valves. The equipment is pushed (most preferable) or driven into the ground, by means of a CPTu rig or similar, and the expansion test is performed every $20 \mathrm{~cm}$. The (basic) pressures required for liftoff the diaphragm $\left(P_{0}\right)$, to deflect $1.1 \mathrm{~mm}$ the centre of the membrane $\left(P_{1}\right)$ and at which the diaphragm returns to its initial position $\left(P_{2}\right.$ or closing pressure) are recorded. Due to the balance of zero pressure measurement method (null method), DMT readings are highly accurate even in extremely soft soils, and at the same time the blade is robust enough to penetrate soft rock or gravel (in the latter, pressure readings are not possible), supporting safely $250 \mathrm{kN}$ of pushing 
force. The test is found especially suitable for sands, silts and clays where the grains are smaller (typically $\frac{1}{10}$ to $\frac{1}{5}$ ) compared to the membrane dimension [8].

Four intermediate parameters, Material Index $\left(I_{D}\right)$, Dilatometer Modulus $\left(E_{D}\right)$, Horizontal Stress Index $\left(K_{D}\right)$ and Pore Pressure Index $\left(U_{D}\right)$, are deduced from the basic pressures $P_{0}, P_{1}$ and $P_{2}$, having some recognizable physical meaning and some engineering usefulness [5], as it will be discussed below. The deduction of current geotechnical soil parameters is obtained from these intermediate parameters covering a wide range of possibilities. In the context of the present work only $E_{D}, I_{D}$ and $K_{D}$ have a physical meaning on the determination of $G_{0}$, so they will be succinctly described as follows [7]:

1. Material Index, $I_{D}$ : Marchetti [5] defined Material Index, $I_{D}$, as the difference between $P_{1}$ and $P_{0}$ basic pressures measured normalized in terms of the effective lift-off pressure. The $I_{D}$ parameter is one of the most valuable indexes deduced from DMT, due to its ability to identify soils throughout a numerical value that can be easily introduced in specific formulae for deriving geotechnical parameters. In a simple form, it could be said that $I_{D}$ is a "fine-content-influence meter" [7], providing the interesting possibility of defining dominant behaviours in mixed soils, usually very difficult to interpret when only grain size is available, thus it may be associate to an index reflecting an engineering behaviour.

2. Horizontal Stress Index, $K_{D}$ : The horizontal stress index [5] was defined to be comparable to the at rest earth pressure coefficient, $K_{0}$, and thus its determination is obtained by the effective lift-off pressure $\left(P_{0}\right)$ normalized by the in-situ effective vertical stress. $K_{D}$ is a very versatile parameter since it provides the basis to assess several soil parameters such as those related with state of stress, stress history and strength, and shows dependency on several factors namely cementation and ageing, relative density, stress cycles and natural overconsolidation resulting from superficial removal, among others. The parameter can be regarded as a $K_{0}$ amplified by penetration effects [5] and displays a typical profile very similar in shape to OCR profiles, giving useful information not only about stress history but also on the presence of cementation structures [7]. Since undrained shear strength of fine soils can be related and obtained via OCR and the relation between $K_{0}$ and angle of shearing resistance is well stated by soil mechanics theories, then the parameter is also used with success in deriving shear strength.

3. Dilatometer Modulus, $E_{D}$ : Stiffness behaviour of soils is generally represented by soil moduli, and thus the base for in-situ data reduction. Stiffness behaviour of soils is generally represented by soil moduli, and thus the base for in-situ data reduction. Theory of Elasticity is used to derive dilatometer modulus, $E_{D}[5]$, by considering that membrane expansion into the surrounding soil can be associated to the loading of a flexible circular area of an elastic half-space, and thus the outward movement of the membrane centre under a normal pressure $\left(P_{1}-P_{0}\right)$ can be calculated. In short, $E_{D}$ is a parameter that includes both Young modulus (E) and Poisson's coefficient $(\nu)$ 
and can be expressed as follows:

$$
E_{D}=\frac{E}{1-\nu^{2}}=34.7\left(P_{1}-P_{0}\right)
$$

Generally speaking, soil moduli depend on stress history, stress and strain levels drainage conditions and stress paths. The more commonly used moduli are constrained modulus $(M)$, drained and undrained compressive Young modulus $\left(E_{0}\right.$ and $\left.E_{u}\right)$ and small-strain shear modulus $\left(G_{0}\right)$, this latter being assumed as purely elastic and associated to dynamic low energy loading.

Maximum shear modulus, $G_{0}$, is indicated by several investigators $[2,7,9]$ as the fundamental parameter of the ground. If properly normalized, with respect to void ratio and effective stress, could be seen as independent of the type of loading, number of loading cycles, strain rate and stress/strain history [9]. It can be accurately deduced through shear wave velocities,

$$
G_{0}=\rho v_{s}^{2}
$$

where $\rho$ stands for density and $v_{s}$ for shear wave velocity.

However, the use of a specific seismic test imply an extra cost, since it can only supply this geotechnical parameter, leaving strength and insitu state of stress information dependent on other tests. Therefore, several attempts to model the maximum shear modulus as a function of DMT intermediate parameters for sedimentary soils have been made in the last decade. Hryciw [10] proposed a methodology for all types of sedimentary soils, developed from indirect method of Hardin \& Blandford [11]. Despite its theoretical base, references on this method are scarce and in general it is not applied for practical analysis due to some scatter around the determination, as illustrated by the results obtained in Portuguese normally consolidated clays [6]. The reasons for this scatter may be related to an expectable deviation of $K_{0}$ due to the important disturbance effects generated by penetration. On the other hand, this methodology ignores dilatometer modulus, $E_{D}$, commonly recognized as a highly accurate stress-strain evaluation, and also lateral stress index, $K_{D}$, and material index, $I_{D}$, which are the main reasons for the accuracy in stiffness evaluation offered by DMT tests [6]. Being so, the most common approaches [12-14] with reasonable results concentrated in correlating directly $G_{0}$ with $E_{D}$ or $M_{D M T}$ (constrained modulus), which have revealed linear correlations with slopes controlled by the type of soil. In 2006, Cruz [6] proposed a generalization of this approach, trying to model the ratio $R_{G} \equiv \frac{G_{0}}{E_{D}}$ as a function of $I_{D}$. In 2008, Marchetti [15] using the commonly accepted fact that maximum shear modulus is influenced by initial density and considering that this is well represented by $K_{D}$, studied the evolution of both $R_{G}$ and $G_{0} / M_{D M T}$ with $K_{D}$ and found different but parallel trends as function of type of soil (that is $I_{D}$ ), recommending the second ratio to be used in deriving $G_{0}$ from DMT, as consequence of a lower scatter. In 2010, using the Theory of Elasticity, Cruz [7] approximate $G_{0}$ as a non-linear function of $I_{D}, E_{D}$ and $K_{D}$, from where a promising median of relative errors close to 0.21 with a mean(std) 
around $0.29(0.28)$ were obtained. It is worth mention that comparing with the previous approach - $R_{G}$ - this approximation, using the same data, lowered the mean and median of relative errors in more than 0.05 maintaining the standard deviation (Table 2).

In this work, to infer about the results quality it will be used some of the same indicators used by Hryciw, Cruz and others that are: the median, the arithmetic mean and standard deviation of the relative errors

$$
\delta_{\widetilde{G_{0}}}^{i}=\frac{\left|\widetilde{G_{0}}(i)-G_{0}(i)\right|}{\left|G_{0}(i)\right|} ; i=1,2, \ldots, N
$$

where $\widetilde{G_{0}}(i)$ stands for the predicted value and $G_{0}(i)$ for the measured value seismic wave velocities (which is assumed to be correct). A final remark to point out that since in this work the no-intercept regression is sometimes used, the $R^{2}$ values will not be presented as they can been meaningfull in this case [16]. It is also worth to remark that in the context of DMT and from the engineering point of view, median is the parameter of choice for assessing the model quality [7] since the final value for maximum shear modulus relies on all set of results obtained in each geotechnical unit or layer.

\section{Data Set, Experiments and Results}

The data set used in the model is exactly the same used in the development of the non-linear $G_{0}$ approximation used by Cruz [7], resulting from 860 DMT measurements performed in Portugal by Cruz and world wide by Marchetti et al. [15] (data kindly granted by Marchetti for this purpose), which included data obtained in all kinds of sedimentary soils, namely clays, silty clays, clayey silts, silts, sandy silts, silty sands and sands. The main statistical measures of $I_{D}, E_{D}$, $K_{D}$ and $G_{0}$ parameters are given in Table 1 as well as in Figure 1.

\begin{tabular}{ccccc}
\hline Values & $I_{D}$ & $E_{D}$ & $K_{D}$ & $G_{0}$ \\
\hline min & 0.0507 & 0.3644 & 0.9576 & 6.4300 \\
$\max$ & 8.8143 & 94.2600 & 20.5000 & 529.2000 \\
median & 0.5700 & 13.4450 & 3.5750 & 77.9100 \\
mean & 0.9134 & 18.8282 & 4.9161 & 92.5165 \\
std & 1.0739 & 18.8264 & 3.6079 & 69.6096 \\
\hline
\end{tabular}

Table 1. Sample statistical measures.

Several types of neural networks (NN) were used in order to improve the results obtained with traditional approaches and to achieve the best results with this kind of tool. The purpose was to find the best NN algorithm for the DMT 

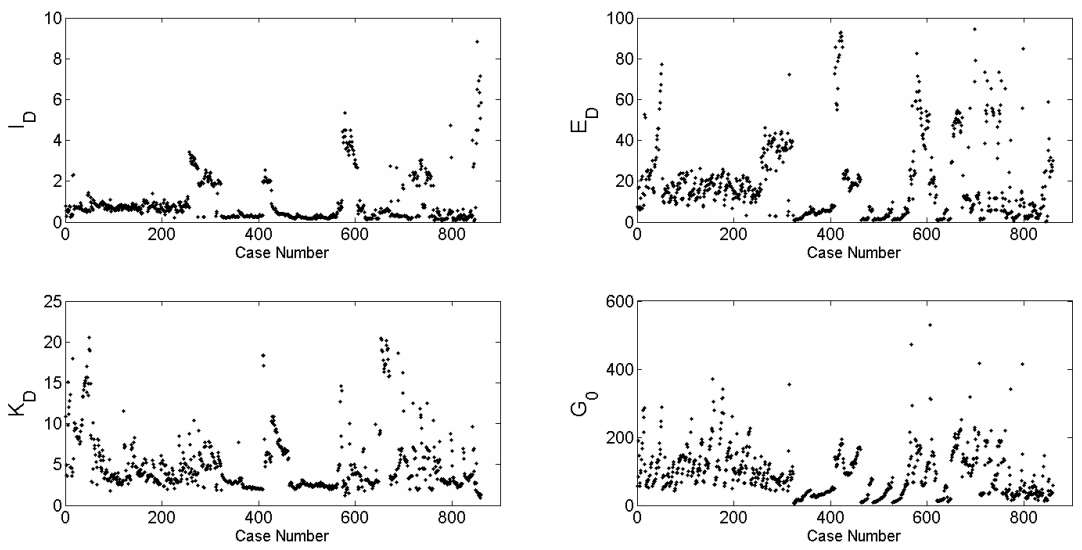

Fig. 1. Sample values for $I_{D}, E_{D}, K_{D}$ and $G_{0}$

problem. This process started by performing some experiments with traditional Multi Layer Perceptrons (MLP's) with different learning algorithms, namely Quasinewton, Conjugated Gradient and Scaled Conjugated Gradient (SCG) but, as it will be shown latter, the results were not as good as expected, and so Radial Basis Function (RBF), Bayesian and Fitting neural networks were then applied. The results obtained with this neural networks were slightly better than those with MLP's but still not significantly better. The last approach was to use Support Vector Regression in order to improve the results with the previous NN. For all the experiments the 10 fold cross validation method with 20 repetitions was used, since this is the most common and widely accepted methodology to guarantee a good neural network generalization. For each NN a huge set of experiments was performed, varying the involved parameters such as the number of neurons in the MLP hidden layer, the number of epochs or the minimum error for stopping criteria. The results presented in Tables 3 to 7 are therefore the best ones for each regression algorithm and represents the mean of the $10 \times 20$ performed tests for each best configuration. It is important to stress the fact that, when compared to traditional approaches where all the data is used to build the model, this methodology tends to produce higher standard deviations since in each experiment only a fraction of the available data is used to evaluate the model.

As stated in Section 2, Cruz [7] was able to improve older results applying non-linear function approximation. In Table 2 the median and mean (standard deviation) of the relative errors obtained by that approaches are presented.

Table 3 shows the best results for the first performed experiments with common MLP regression using three different learning algorithms: Quasinewton, Conjugated Gradient and SCG. It can be seen that there was a small improvement in the results when compared to those of Table 2 but not very significant. 


\begin{tabular}{lcc}
\hline & Type & Median/Mean(std) \\
\hline Non-Linear & $G_{0}=\alpha E_{D}\left(I_{D}\right)^{\beta}$ & $0.28 / 0.34(0.29)$ \\
Regression & $G_{0}=E_{D}+E_{D} e^{\left(\alpha+\beta I_{D}+\gamma \log \left(K_{D}\right)\right)}$ & $0.21 / 0.29(0.28)$ \\
\hline
\end{tabular}

Table 2. The results obtained with non-linear regression [7].

\begin{tabular}{cccc}
\hline & Type & $\begin{array}{c}\text { Hidden } \\
\text { neurons }\end{array}$ & $G_{0}=f\left(I_{D}, E_{D}, K_{D}\right)$ \\
\hline \multirow{4}{*}{ NN } & Quasinewton & 50 & $0.20 / 0.38(0.72)$ \\
& Conj.Grad. & 100 & $0.19 / 0.30(0.38)$ \\
& SCG & 40 & $0.20 / 0.28(0.33)$ \\
\hline
\end{tabular}

Table 3. The results (Median/Mean(std)) obtained with Multi Layer Perceptrons.

The second set of experiments was performed using Bayesian, RBF's (with thin plate spline activation function) and Fitting neural networks regression, by applying several combinations for the number of iterations in RBF's or the number of inner and outer loops for the Bayesian NN's. The best obtained results with these algorithms are presented in Table 4.

\begin{tabular}{cccc}
\hline & Type & $\begin{array}{c}\text { Hidden } \\
\text { neurons }\end{array}$ & $G_{0}=f\left(I_{D}, E_{D}, K_{D}\right)$ \\
\hline \multirow{4}{*}{ NN } & MLP-Bayesian & 20 & $0.20 / 0.29(0.30)$ \\
& RBF & 200 & $0.20 / 0.31(0.39)$ \\
& Fitting & 60 & $0.17 / 0.27(0.29)$ \\
\hline
\end{tabular}

Table 4. The results (Median/Mean(std)) obtained with Bayesian, RBF and Fitting neural networks.

These results are not significantly better than those obtained with common MLP's, with the exception of the fitting neural network that shows a significant improvement when compared with the others and also with traditional approaches.

An attempt to improve the quality of results was carried out by using Support Vector Regression (SVR). Support Vector Machines [17] are based on the statistical learning theory from Vapnik and are specially suited for classification. However, there are also algorithms based in the same approach for regression problems known as Support Vector Regression (SVR). Two different kinds of 
SVR algorithms: $\epsilon$-SVR, from Vapnik [18] and $\nu$-SVR from Schölkopf [19] were applied, which differ in the fact that $\nu$-SVR uses an extra parameter $\nu \in(0,1]$ to control the number of support vectors. For these experiments different values for the parameter $C$ (cost) and for parameters $\epsilon$ and $\nu$ were used.

The best results obtained with both $\epsilon$-SVR and $\nu$-SVR are shown in Table 5 reveling slightly better results when compared with those obtained with the fitting neural network and better than those obtained with MLP's and the traditional regression algorithms.

\begin{tabular}{clc}
\hline Type & Cost $/ \epsilon(\nu)$ & $G_{0}=f\left(I_{D}, E_{D}, K_{D}\right)$ \\
\hline$\epsilon$-SVR & $200 / 0.1$ & $0.16 / 0.27(0.43)$ \\
$\nu$-SVR & $200 / 0.8$ & $0.16 / 0.27(0.41)$ \\
\hline
\end{tabular}

Table 5. The results (Median/Mean(std)) obtained with Support Vector Regression.

In all the previous experiments it was tried to estimate $G_{0}$ as a function of $I_{D}, E_{D}$ and $K_{D}$. Following the same strategy as Cruz [7], other experiments were performed which results are presented in Table 6 . In these experiments $G_{0}$ was estimated only as a function of two of the three available variables. As last option $E_{D}$ was used as a single input variable but the results were worst than those obtained using more input variables (due to lack of space they are not shown here). In these experiments only SVR's were used, as they presented the best results in the previous experiments.

\begin{tabular}{cccc}
\hline Type & $G_{0}=f\left(E_{D}, K_{D}\right)$ & $G_{0}=f\left(I_{D}, E_{D}\right)$ & $G_{0}=f\left(I_{D}, K_{D}\right)$ \\
\hline$\epsilon$-SVR & $0.19 / 0.32(0.46)$ & $0.20 / 0.32(0.42)$ & $0.29 / 0.55(0.88)$ \\
$\nu$-SVR & $0.19 / 0.32(0.47)$ & $0.20 / 0.32(0.44)$ & $0.30 / 0.55(0.96)$ \\
\hline
\end{tabular}

Table 6. The results (Median/Mean(std)) obtained with Support Vector Regression using only two input variables.

After the last set of experiments it can be seen that the best results are still those presented in Table 5 , the ones that were obtained by using the three available parameters $K_{D}, I_{D}$ and $E_{D}$.

The final experiments were conducted splitting $I_{D}$ values in coherent groups, namely those related with clay, silt and sandy soils, respectively represented by the following $I_{D}$ intervals: $I_{D}<0.6,0.6 \leq I_{D}<1.8$ and $I_{D} \geq 1.8$. This operation made a partition of the original 860 data set elements in subsets with 449,259 
and 152 elements respectively. Experiments with these data sets (subsets) were performed using only SVR algorithms since they were the ones with better results in the previous experiments. Results are presented in Table 7.

\begin{tabular}{ccc}
\hline & Subsets & $G_{0}=f\left(I_{D}, E_{D}, K_{D}\right)$ \\
\hline & $I_{D}<0.6$ & $0.16 / 0.29(0.41)$ \\
$\epsilon$-SVR & $0.6 \leq I_{D}<1.8$ & $0.15 / 0.24(0.35)$ \\
& $I_{D} \geq 1.8$ & $0.15 / 0.39(0.57)$ \\
\hline \multirow{3}{*}{$\nu$-SVR } & $I_{D}<0.6$ & $0.17 / 0.29(0.37)$ \\
& $0.6 \leq I_{D}<1.8$ & $0.15 / 0.24(0.38)$ \\
& $I_{D} \geq 1.8$ & $0.16 / 0.37(0.60)$ \\
\hline
\end{tabular}

Table 7. The results (median/mean(std)) obtained using the subsets of the original data set and for the estimation of $G_{0}$ as a function of $I_{D}, E_{D}$ and $K_{D}$.

Although these final results can be pointed as very interesting, specially with $\epsilon$-SVR, it can not be concluded that these are better than those obtained with the complete data set (Table 5) once there are one larger subset with a result slightly higher and two smaller subsets with a slightly lower result (remember $I_{D}$-values distribution in Figure 1$)$. This indicates that the methodology can produce interesting results in some particular cases, deserving a more detailed study. Nevertheless from a geotechnical point of view it may be considered a very promising result.

\section{Conclusions}

A new approach was applied to predict maximum shear modulus by DMT using Neural Networks. Based on performed experiments it is possible to outline the following considerations:

- Neural Networks improve the current state-of-the-art in terms of $G_{0}$ prediction through DMT intermediate parameters.

- The results show that, in general, NN lead us to much smaller medians, equivalent means and higher standard deviations in respect to relative errors, when compared to traditional approaches.

- Regarding the problem characteristics the SVR approach gives the best results considering the median as the main quality measure as discussed earlier.

- The Fitting NN seems to be the most robust in terms of the three quality control parameters used, improving the results of traditional approaches.

- The unbalanced data distribution, regarding the $I_{D}$ partition, postpone a final conclusion about the improvement of model quality to the availability of a more balanced sample. 


\section{References}

1. C. Clayton and G. Heymann. Stiffness of geomaterials at very small strains. Géotechnique, 51(3):245-255, 2001.

2. M. Fahey. Soil stiffness values for foundation settlement analysis. In Proc. 2nd Int. Conf. on Pre-failure Deformation Characteristics of Geomaterials, volume 2, pages 1325-1332. Balkema, Lisse, 2001.

3. Ralph B. Peck, Walter E. Hanson, and Thomas H. Thornburn. Foundation Engineering (2nd Edition). John Wiley \& Sons, 1974.

4. T. Lunne, P. Robertson and J. Powell. Cone penetration testing in geotechnical practice. Spon E \& F N, 1997.

5. S. Marchetti. In-situ tests by flat dilatometer. Journal of the Geotechn. Engineering Division, 106(GT3):299-321, 1980.

6. N. Cruz, M. Devincenzi, and A. Viana da Fonseca. Dmt experience in iberian transported soils. In Proc. 2nd International Flat Dilatometer Conference, pages 198-204, 2006.

7. N. Cruz. Modelling geomechanics of residual soils by DMT tests. PhD thesis, Universidade do Porto, 2010.

8. S. Marchetti. The flat dilatometer: Design applications. In Third Geotechnical Engineering. Conf. Cairo University, 1997.

9. P. W. Mayne. Interrelationships of dmt and cpt in soft clays. In Proc. 2nd International Flat Dilatometer Conference, pages 231-236, 2006.

10. R.D. Hryciw. Small-strain-shear modulus of soil by dilatometer. Journal of Geotechnical Eng. ASCE, 116(11):1700-1716, 1990.

11. B.O. Hardin and G.E. Blandford. Elasticity of particulate materials. J. Geot. Eng. Div., 115(GT6):788-805, 1989.

12. B.M. Jamiolkowski, C.C. Ladd, J.T. Jermaine, and R. Lancelota. New developments in field and laboratory testing of soilsladd, c.c. In XI ISCMFE, volume 1, pages $57-153,1985$.

13. J.P. Sully and R.G. Campanella. Correlation of maximum shear modulus with dmt test results in sand. In Proc. XII ICSMFE, pages 339-343, 1989.

14. H. Tanaka and M. Tanaka. Characterization of sandy soils using CPT and DMT. Soils and Foundations, 38(3):55-65, 1998.

15. S. Marchetti, P. Monaco, G. Totani, and D. Marchetti. From Research to Practice in Geotechnical EngineeringD.K. Crapps, volume 180, chapter In -situ tests by seismic dilatometer (SDMT), pages 292-311. ASCE Geotech. Spec. Publ., 2008.

16. Yufen Huang and Norman R. Draper Transformations, regression geometry and R2, Computational Statistics \& Data Analysis, 42(4), pages 647-664, 2003

17. C. Cortes and V. Vapnik. Support-vector Networks. Journal of Machine Learning, 20(3), pages 273-297, 1995.

18. V. Vapnik. Statistical Learning Theory. Wiley, New York, NY, 1998.

19. B. Schölkopf, A. Smola, R. C. Williamson, and P. L. Bartlett. New support vector algorithms. Neural Computation, 12, pages 1207-1245, 2000. 